\title{
Assesment of Radial Artery by Means of Doppler Ultrasonography and Soft Tissue Roentgenogram in End-Stage Renal Disease Patients
}

\author{
Ahmet Feyzi Abacilar ${ }^{1 *}$, Umit Duman², Omer Faruk Dogan ${ }^{3}$ \\ ${ }^{1}$ Izmir Sifa Hospital, Sanayi Caddesi No. 7, Bornova, İzmir, Turkey \\ ${ }^{2}$ İstanbul American Hospital, Department of Cardiovascular Surgery, İstanbul, Turkey \\ ${ }^{3}$ Adana Numune Education and Training Hospital, Department of Cardiovascular Surgery, Adana, Turkey \\ Email: afabacilar@hotmail.com
}

Received July 4, 2013; revised August 5, 2013; accepted August 13, 2013

Copyright (c) 2014 Ahmet Feyzi Abacilar et al. This is an open access article distributed under the Creative Commons Attribution License, which permits unrestricted use, distribution, and reproduction in any medium, provided the original work is properly cited. In accordance of the Creative Commons Attribution License all Copyrights (C) 2014 are reserved for SCIRP and the owner of the intellectual property Ahmet Feyzi Abacilar et al. All Copyright (C 2014 are guarded by law and by SCIRP as a guardian.

\section{ABSTRACT}

Background: Although the radial artery (RA) is mostly selected for the creating of arterio-venous fistula (AVF) in end-stage renal disease patients (ESRDP), it still represents a suitable site to simply assess systemic calcification. Our goal of this study was to research the RA before the arteriovenous fistula creation in ESRDP and evaluate the risk factor determinants. Material and Methods: The RA has been determined to estimate vascular calcification in 67 ESRDPs on dialysis by doppler ultrasonography (USG). If there was any pathologic conditions, direct examination of the forehand soft tissue roentgenograms as a simply method was made. Preoperative spectral Doppler sonography measured radial artery peak systolic velocity during tight fist clenching for $\mathbf{3}$ minutes and after fist relaxation. Vessel diameters and peak systolic velocity were assessed for predictive value based on subsequent fistula adequacy. RA calcification was scored from Grade 0 which was defined as no calcification to Grade 4 which was described as severe calcification from proximal to distal end of RA. Results: Doppler USG has shown that the major or minor RA calcification was detected in 11 patients (16.5\%). In these patients, direct roentgenogram also demonstrated RA calcific disease. Total and LDL cholesterol levels, gender and smoking status have no influence on calcification scores. When the dialysis periods are concerned, there was no correlation with the RA calcification. Serum calcium and phosphate levels, rather than calcium-phosphate products, and age were correlated with high calcification scores. The highest prevalence of RA calcification was also found in diabetic group. Conclusion: Our data indicate that RA calcification is highly prevalent among ESRDP, with the highest prevalence among diabetics. The RA was used as an A-V fistual creation in ESRDPs, which may be risky since the calcification percentage is higher. Therefore, a simple roentgenographic survey may help to identify patients with severe calcified RA. Because our roengenographyc results were correlated with doppler USG, it can be used to determine RA calcific disease before the arteriovenous fistula creation.

\section{KEYWORDS}

Radial Artery; Calcification; End-Stage Renal Failure Patients

\section{Introduction}

The radial artery plays an important role as an AVF creation in the cases of ESRDP. Thus, preoperative RA investigation for AVF by the use of rutine technique is necessary. To date, frequently, tests such as doppler ultraso-

\footnotetext{
${ }^{*}$ Corresponding author.
}

nography, Allen or modified-Allen tests are used as screening methods for assessing the hand circulation in most centers. However, these above technical procedures cannot be used routinely and most of them are not costeffective. It is known that, ASRDPs having chronic dialysis, an intimal calcification develops on the vascular wall rather than a calcification originating from the endo- 
thelium. Because of the use of hand and forearm X-ray films taken in soft tissue density, it is easy and quite reliable that we compared our results of doppler USG and simple roengenographic investigation in our ESRDPs.

\section{Materials and Methods}

We analysed the results of doppler USG and soft tissue roentgenograms of the RA in 67 ESRDPs before AVF creation. The ages of the patients varied between 20 and 76 years (the mean age of $49 \pm 2.3$ years). The mean age of the patients was 48.84 with the Standard Deviation (SD) of 14,841 years. 28 cases were male. Number of patients have underwent hemodialysis more than five years. 12 cases were previously hospitalised because they had coronary artery symptoms. RA calcification was recorded in older cases (more than 60 years).

Before the roentgenographic examination, for investigation of the RA Allen and Modified Allen test was made by the same surgeon. Our results were negative in all patients. We used doppler USG for determining the radial artery calcification to estimate the vascular calcification. The anteroposterior diameter of the radial artery is measured in the transverse dimension on gray scale sonography. Spectral Doppler evaluation of the radial artery was performed. An initial spectral Doppler image of the distal radial artery at the wrist was obtained after the patient maintained a tightly clenched fist for nearly 3 minutes. After 3 minutes, the fist was relaxed, and additional spectral Doppler imaging of the artery was performed. Peak systolic velocity and end-diastolic velocity of the RA were measured in a representative waveform in each patient. The resistive index was a measure of the downstream flow resistance and was calculated as the difference between the respective measurements obtained after fist release (post) and during fist clenching (pre).

When there was an evidence for calcification and/or narrowing of the artery due to calcified plaque, the simple direct roentgenogram of the hand and forearm in soft tissue density as an alternative diagnostic tool was also used. We scored the calcification status from Grade 0 (no calcification) to Grade 4 (severe calcification, from proximal to distal end of both radial and unlar artery) by detailed measurement of the calcification through the radial artery by the use of doppler USG. We investigated for the clinical significancy of sex, age, serum calcium (Ca) and phosphate levels, smoking status, peripheral artery occlusive disease (known or unknown), carotid artery stenotic disease (known), presence of hypercholesterolemia including LDL levels.

\section{Statistical Analysis}

For the statistical analysis, SPSS 13.0 for Windows was used. For evaluation of the relationship of serum calcium and phosphate levels and radial artery calcification, independent Samples t-test were used. To find out the relationship between radial artery calcification and sex, age, serum $\mathrm{Ca}$ and phosphate levels, smoking status, peripheral artery occlusive disease (known or unknown), carotid artery stenotic disease (known), prensence of hypercholesterolemia, serum levels of total cholesterol and LDL levels chi-square test was used.

\section{Results}

Since the study was first designed to be a cross-sectional study to find out the frequency of radial artey calcification in ESRDPs a wide range of patients were included in the study which soon led us to divide the patients into specific age groups. Of whole patients 11 (16.4\%) were identified to have grade 1 to 4 radial artery calcification by doppler USG and after X-ray films (Figures 1-4) ; in 2 females and in 9 males. In all patients, the results of doppler USG and direct roengenograms were correlated. Peak systolic velocity and end-diastolic velocity of the RA were high in the 11 patients. We obtained direct roengenograms in the patients. Patient with severely calcified RA which was detected using a doppler USG, were mainly typical (Figures 3 and 4). Interestingly, there was no evidence of RA occlusion when we tested the RA with the use of Allen test.

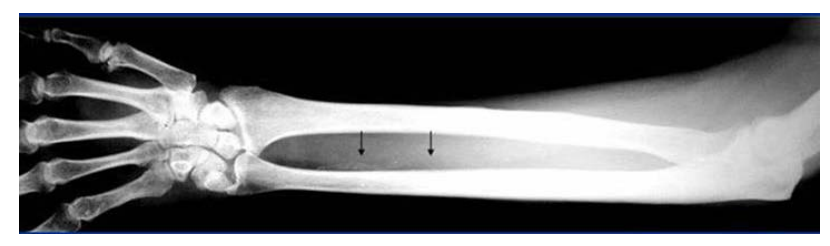

Figure 1. Heavily calcification is demonstrating in this patient with insuline dependent diabetes mellitus. Arrow heads is showing that calcific radial and the digital artery (Grade 1).

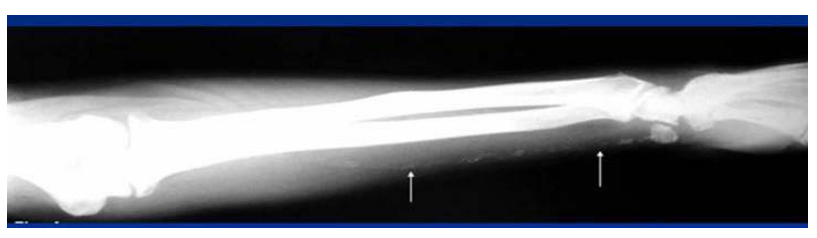

Figure 2. Only the ulnar artery calcific changes is obtaining from the patient without diabetes mellitus. No evidence of radial artery calcification in this figure.

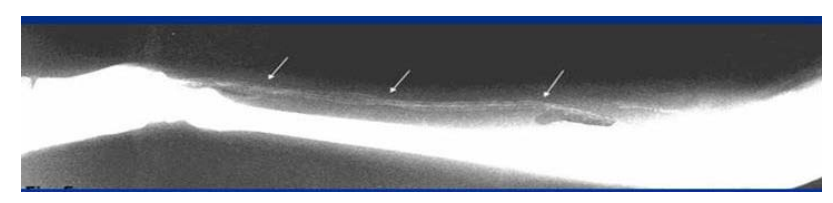

Figure 3. Gradee 3 diffuse radial artery calcification is showing. Calcific changes were showed that about $2 / 3$ distal part of the radial artery. 


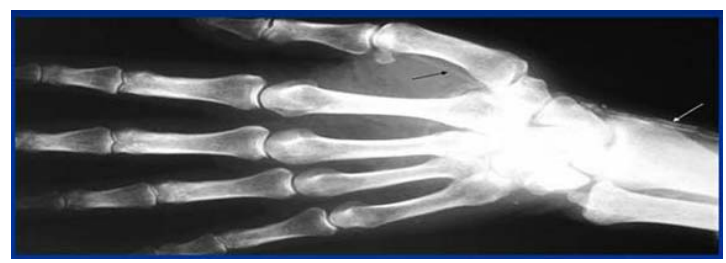

Figure 4. Grade 4 calcific score is showing in patient with diabetes mellitus. Both of heavily diseased upper limb arteries is demonstrating by means of soft tissue roentgenogram. The radial and the unlar arteries calcification is seen from distal part to proxymal.

The gender was not found to be a statistically significant risk factor for radial artery calcification in this study $(p>0.05)$ (Table 1). When the age groups are explored, we found that with the increasing age, the percentage of the radial artery calcification was also increasing. The variation of the age groups and its realationship the radial artery calcification are explored in Table 2. Among 67 patients, 6 were known to have diabetes mellitus (DM) (all undergoing insulin treatment). The renal pathology of this patient group was nephropathy caused by the primary disease which is DM itself. Of those 6 diabetic patients $3(50 \%)$ were identified to have radial artery calcification. DM has recorded as a major risk factor for radial artery calcification in ESRDPs undergoing dialysis $(p<0.05)$ (Table 3). In this study, none of the patients had carotid artery stenotic disease. Thus, no statistical anaylsis could have been recorded using this parameter whereas peripheral artery occlusive disease was identified in 2 of the patients whom at the same time had radial artery calcification. The statistical analysis revealed a significant correlation between the peripheral artery occlusive disease and radial artery calcification $(\mathrm{p}<0.05)$ (Table 4).

When the serum calcium and phosphate levels have evaluated among the calcification positive and negative groups, a statistically significant correlation was found (p $<0.05$ ) (Table 5); however, there was no relation between the calcium and phosphate products (CaxP) (p > 0.05). We also analyzed the relationship between the presence of hypercholesterolemia and the radial artery calcification. Hypercholesterolemia was not found to be a statistically significant risk factor for radial artery calcification ( $p>0.05)$. The serum cholesterol and LDL levels of the patients were also explored. There was again no relationship between these parameters and radial artery calcification ( $>0.05$ ) (See Tables 6 and 7). In addition, smoking history was not found to be a significant risk factor for radial artery calcification in both groups ( $p>0.05$ ) (See Table 8).

\section{Discussion}

The radial artery is the most common site for the crea-
Table 1. Sex vs. radial artery calcification.

\begin{tabular}{cccc}
\hline Sex & Calcification(+) & Calcification(-) & Total \\
\hline Female & 2 & 26 & 28 \\
\% within sex & $7.1 \%$ & $92.9 \%$ & $100.0 \%$ \\
\% within RAC & $18.2 \%$ & $26.4 \%$ & $41.8 \%$ \\
Male & 9 & 30 & 39 \\
\% within sex & $23.1 \%$ & $76.9 \%$ & $100.0 \%$ \\
\% within RAC & $81.8 \%$ & $53.6 \%$ & $58.2 \%$ \\
Total & 11 & 56 & 67 \\
\% within sex & $16.4 \%$ & $83.6 \%$ & $100.0 \%$ \\
\% within RAC & $100.0 \%$ & $100.0 \%$ & $100.0 \%$ \\
\hline
\end{tabular}

RAC: Radial artery calcification.

Table 2. The age groups vs. radial artery calcification.

\begin{tabular}{cccc}
\hline Age Groups & Calcification(+) & Calcification(-) & Total \\
\hline 20 - 39 & 1 & 14 & 15 \\
\% within age & $6.7 \%$ & $93.3 \%$ & $100.0 \%$ \\
\% within RAC & $9.1 \%$ & $25.0 \%$ & $22.4 \%$ \\
40 - 49 & 1 & 15 & 16 \\
\% within age & $6.3 \%$ & $93.8 \%$ & $100.0 \%$ \\
\% within RAC & $9.1 \%$ & $26.8 \%$ & $23.9 \%$ \\
50 - 59 & 2 & 12 & 14 \\
\% within age & $14.3 \%$ & $85.7 \%$ & $100.0 \%$ \\
\% within RAC & $18.2 \%$ & $21.4 \%$ & $20.9 \%$ \\
z60 & 7 & 15 & 22 \\
\% within age & $31.8 \%$ & $68.2 \%$ & $100.0 \%$ \\
\% within RAC & $63.6 \%$ & $26.8 \%$ & $32.8 \%$ \\
Total & 11 & 56 & 67 \\
\% within age & $16.4 \%$ & $83.6 \%$ & $100.0 \%$ \\
\%within RAC & $100.0 \%$ & $100.0 \%$ & $100.0 \%$ \\
\hline
\end{tabular}

RAC: Radial artery calcification.

Table 3. Diabetes mellitus (DM) vs. radial artery calcification.

\begin{tabular}{cccc}
\hline DM & Calcification(+) & Calcification(-) & Total \\
\hline Positive & 6 & 6 & 12 \\
\% within DM & $50.0 \%$ & $50.0 \%$ & $100.0 \%$ \\
\% within RAC & $54.5 \%$ & $10.7 \%$ & $17.9 \%$ \\
Negative & 5 & 50 & 55 \\
\% within DM & $9.1 \%$ & $90.9 \%$ & $100.0 \%$ \\
\% within RAC & $45.5 \%$ & $89.3 \%$ & $82.1 \%$ \\
Total & 11 & 56 & 67 \\
\% within DM & $16.4 \%$ & $83.6 \%$ & $100.0 \%$ \\
\% within RAC & $100.0 \%$ & $100.0 \%$ & $100.0 \%$ \\
\hline
\end{tabular}

RAC: Radial artery calcification.

tion of arterio-venous fistula in end-stage renal disease patients. RA is also used in coronary artery bypass surgery as an arterial conduit. High quality clinical and angiographic results has previously been reported by the most authors [1-4].

With the resurgence of the use of the radial artery for coronary artery bypass grafting or fistula creation in ESRDP, tests such as doppler ultrasonography, Allen or modified Allen test have been used as screening tests for 
assessing the hand circulation, and the benefits are also recorded [5,6]. But, diffuse calcified radial artery in case with coronary artery disease have been reported by Dogan et al. using computed tomography of the forearm before the radial artery harvesting in CABG patients for the first time [7].

In our study, because of this method is cheapest and easy to use and simple we used forearm X-ray films for evaluation of radial artery calcific disease in largest patient who diagnosed ESRDP and high incidence of peripheral artery calcification was recorded in this groups. We sough to determine the incidence of radial artery calcification in ESRDPs using direct roentgenogram of the forearm to establish its use in evaluation of the radial artery. Also we established the high rate of radial artery calcification in ESRDPs with DM.

Nicolasi et collegues have examined the radial artery calcification in diabetic and non-diabetic patients by Doppler ultrasonography [8]. According to our cases, they reported that both incidence and severity of the calcification has increased in the diabetic group [8]. They have accounted RA calcification index for calcification density, longitidunal vessel involvement and bilaterality. One of the drawbacks of using this test is that there are no established standard criteria to differentiate normal or abnormal dopper ultrasound results. The advantage of the Doppler is that it can show the upper limb arterial system anatomy and measure the flow velocity; however, the criteria for evaluation vary considerably. The most important disadvantage is the operator dependence and subjectivity of this technique. In addition, it can not be routinely used for evaluation of the upper extremity arterial system. Herein, we reported that among 67 ESRD patients, 6 were diabetic who treated with insulin treatment. Of those 3/6 (50\%) had radial artery calcification. We established DM as a main risk factor for radial artery calcification in ESRDP undergoing dialysis. According to our findings, if ESRDP or CABG candidates have insuline dependent diabetes mellitus in preoperative pe- riod for the creation A-V fistula or the choise of coronary bypass conduit soft tissue roengenogram should be decide in these particular cases.

The modified-Allen test is a subjective operator/patient dependent test, and there have been a number of reports of unexpected hand ischemia after radial artery intervention, including radial artery hervesting [9]. On the other hand, the Allen test is a simple and cost-effective test, however there is still some debate about whether it is a valid screening test. Interestingly, Allen and modified-Allen test results were found as negative in our patients with radial artery calcification. We believe that these tests do not give reliable information about the quality of radial artery in some patients, but our Allen test findings may be resulting from enough blood supply to the hand from ulnar artery.

Shigematsu et al. reported phosphate overload could accelerate the vascular calcium deposition in ESRD pa-

Table 4. Peripheral artery occlusive disease (PAOD) vs. radial artery calcification.

\begin{tabular}{cccc}
\hline $\begin{array}{c}\text { Peripheral Artery } \\
\text { Occlusive Disease }\end{array}$ & Calcification(+) & Calcification(-) & Total \\
\hline Positive & 2 & & 2 \\
\% within PAOD & $100.0 \%$ & - & $100.0 \%$ \\
\% within RAC & $18.2 \%$ & & $3.0 \%$ \\
Negative & 9 & 56 & 65 \\
\% within PAOD & $13.8 \%$ & $86.2 \%$ & $100.0 \%$ \\
\% within RAC & $81.8 \%$ & $100.0 \%$ & $97.0 \%$ \\
Total & 11 & 56 & 67 \\
\% within PAOD & $16.4 \%$ & $83.6 \%$ & $100.0 \%$ \\
\% within RAC & $100.0 \%$ & $100.0 \%$ & $100.0 \%$ \\
\hline
\end{tabular}

RAC: Radial artery calcification.

Table 5. Serum calcium (CA) and phosphate (P) levels.

\begin{tabular}{ccc}
\hline & Calcification $(+)$ & Calcification( $(-)$ \\
\hline Ca $($ Mean \pm SD) & $9.8455 \pm 0.97094$ & $10.4818 \pm 0.69321$ \\
$\mathrm{P}($ Mean \pm SD) & $4.1000 \pm 1.35425$ & $3.2964 \pm 0.88687$ \\
\hline
\end{tabular}

Table 6. Hypercholesterolemia vs. radial artery calcification.

\begin{tabular}{|c|c|c|c|}
\hline Hypercholesterolemia & Calcification(+) & Calcification(-) & Total \\
\hline \multirow{2}{*}{$\begin{array}{c}\text { Positive } \\
\text { \% within hypercholesterolemia } \\
\text { \% within RAC }\end{array}$} & $\begin{array}{c}1 \\
12.5 \%\end{array}$ & $\begin{array}{c}7 \\
87.5 \%\end{array}$ & $\begin{array}{c}8 \\
100.0 \%\end{array}$ \\
\hline & $9.1 \%$ & $12.5 \%$ & $11.9 \%$ \\
\hline \multirow{2}{*}{$\begin{array}{c}\text { Negative } \\
\text { \% within hypercholesterolemia } \\
\text { \% within RAC }\end{array}$} & $\begin{array}{c}10 \\
16.9 \%\end{array}$ & $\begin{array}{c}49 \\
83.1 \%\end{array}$ & $\begin{array}{c}59 \\
100.0 \%\end{array}$ \\
\hline & $90.9 \%$ & $87.5 \%$ & $89,1 \%$ \\
\hline \multirow{2}{*}{$\begin{array}{c}\text { Total } \\
\text { \% within hypercholesterolemia } \\
\text { \% within RAC }\end{array}$} & $\begin{array}{c}11 \\
16.4 \%\end{array}$ & $\begin{array}{c}56 \\
83.6 \%\end{array}$ & $\begin{array}{c}67 \\
100.0 \%\end{array}$ \\
\hline & $100.0 \%$ & $100.0 \%$ & $100.0 \%$ \\
\hline
\end{tabular}

RAC: Radial artery calcification. 
Table 7. Serum total cholesterol and LDL levels.

\begin{tabular}{ccc}
\hline & Calcification(+) & Calcification(-) \\
\hline $\begin{array}{c}\text { Total Cholesterol } \\
(\text { Mean } \pm \text { SD) }\end{array}$ & $144.73 \pm 31.544$ & $154.27 \pm 40.338$ \\
LDL $($ Mean \pm SD) & $76.18 \pm 28.740$ & $84.86 \pm 29.444$ \\
\hline
\end{tabular}

Table 8. Smoking status vs. radial artery calcification.

\begin{tabular}{cccc}
\hline Smoking Status & Calcification(+) & Calcification(-) & Total \\
\hline $\begin{array}{c}\text { Smoking } \\
\text { \% within }\end{array}$ & 1 & 9 & 10 \\
$\begin{array}{c}\text { Smoking Status } \\
\text { \% within RAC }\end{array}$ & $10.0 \%$ & $90,0 \%$ & $100.0 \%$ \\
Not Smoking & 10 & $16.1 \%$ & $14.9 \%$ \\
\% within & $17.5 \%$ & 47 & 57 \\
$\begin{array}{c}\text { Smoking Status, } \\
\text { \% within RAC }\end{array}$ & $90.9 \%$ & $82.5 \%$ & $100.0 \%$ \\
$\quad$ Total & 11 & $83.9 \%$ & $85.1 \%$ \\
\% within & $16.4 \%$ & 56 & 67 \\
$\begin{array}{c}\text { Smoking Status } \\
\text { \% within RAC }\end{array}$ & $100.0 \%$ & $100.0 \%$ & $100.0 \%$ \\
\hline
\end{tabular}

RAC: Radial artery calcification.

tients [10]. When they harvested the radial artery they found that in the extracellular matrix there is a high affinity for calcium deposition in the high phosphate medium [10]. Furthermore, most authors reported risk factors for radial artery calcification such as DM, chronic renal failure, aortofemoral occlusive disease and age with the use of Doppler Ultrasonography $[8,10,11]$. KaneToddhall et al. have showed that there is an increased prevalence of mild intimal thickening, medial sclerosis and medial calcification in radial arteries compared with internal mammary artery [12]. Based on these findings, we think that more reliable simple radiologic methods should be used in the preoperative period for evaluation of the RA especially in patients at risk for calcification such as in patients with high risk radial artery calcification in order to do more reliable radial artery harvesting or A-V fistula creation. It is known that in ESRDP an intimal calcification develops on the vascular wall rather than a calcification originating in the endothelium [10] and cardiovascular disease is a major problem in these patients with calcification being one of the conspicuous feature of the atherosclerotic vessels $[10,13]$. According to the literature, we found that the calcification along the wall of the radial and the ulnar artery in some patients. However, Davies et al. have reported that vascular calcium deposits occured more in intima than the from arteral wall [14].

In the present study, we presented our investigation of factors affecting the radial artery calcification by means of simple soft tissue direct roentgenographic examination of the fore arm in ESRDP. Our study clearly shows that in ESRDP radial artery should be investigated by soft tissue density roengenogram as an additional method and should be examine more carefully than the normal population. We believe that the use of radial artery grafts in ESRDP for creating A-V fistulas is a provoking clinical controversy since a considerably high ratio (17\%) of radial artery calcification is reported. We also suggest that in these patient groups a preoperative evaluation of radial artery should be perform using a hand and forearm X-ray films taken in soft tissue density which is easy to use and quite reliable. The routine use of this method can also be helpful in patients with DM. Shoul keep in mind, the radial artery calcification can be seen after CABG operation especially in cases with insuline dependent DM when the patient had high level of $\mathrm{Ca}$ and phosphate production. Serum calcium and phosphate levels, but not calcium-phosphate products, were correlated with calcification scores According to these findings, we hope that high Calcium and Phosphate level additionally insuline dependent DM seems to be the most important role than others risk factors. The other technical approach is conventional angiography which is still the gold standard for evaluation of arterial system anatomy and pathology, but has several disadvantages including long procedure time, needing the contrast medium, arterial catheterization and potential complications such as arterial occlusion and dissection. On the other side, sometimes angiography is not give the inform about the arterial wall properties. Therefore, routine use of conventional angiography for radial artery evaluation is not practical and time consuming method.

Olsson et collegues have indicated that overall calcium phosphorus depositions in stenotic arteriovenous fistulas (AVF) in another study [15]. In this investigation the vascular samples have been obtained from patients with ESRD and they have determined the occurrence of calcium depositions in AVF. They had measured calcium and phosphorus content by use of scanning electron microscopy. They have reported the calcium phosphate deposition was differe from calcium compounds than from atherosclerotic samples.

Higly occlusion rates of AVF were reported in ESRD patients in the literature [16,17]. Gade et al. have reported that the overall patency rate for AVF used for haemodialysis was 50\% after one year and 38\% after two years in their study after arterio-venous fistula prosedure [16]. They have suggested that the upper arm arteriovenous fistula can serve as a second choice, when a radio-cephalic fistula fails. The results of this investigation are supported our study. We suggest when the distal part of arterial calcification is found we propose the AVF creation may perform on the upper level of the limb (in Grade 1 or 2 radial artery calcification).

\section{Conclusion}

In conclusion, we suggest using soft tissue density roentgenograms of the forearm in evaluation of radial ar- 
tery in ESRDP with DM and/or high level of plasma calcium and phosphate production especially in preoperative period routinely (preoperative arterio-venous fistula creation and/or coronary artery bypass conduit). For the first time, we also established a Grade 0 to 4 calcification score in the upper arm in these particular patients. We recorded that four patients had Grade 1, three patients had grade 2, two patients had grade 3 and the remaining 2 patients had grade 4 radial artery calcific disease. Our investigations show that the radial artery calcific changes can start from distal parts to the proxymal side. But we believe that although the radial artery is not appropriate for CABG in ESRD patients, it represents a suitable site to simply assess systemic calcification. Moreover, RA is the site for creating arteriovenous fistula, i.e., life line for hemodialysis patients. The routine use of this simple, cost-effective and reliable method can be helpful for preoperative evaluation of the radial artery.

\section{REFERENCES}

[1] C. Acar, A. Ramsheyi, J. Pagny, V. Jebera, P. Barrier, J. Fabriani, A. Deloche, J. Guermonprez and A. Carpentier, “The Radial Artery for Coronary Artery Bypass Grafting. Clinical and Angiographic Results at Five Years,” The Journal of Thoracic and Cardiovascular Surgery, Vol. 116, No. 6, 1998, pp. 981-989. http://dx.doi.org/10.1016/S0022-5223(98)70050-9

[2] A. H. Chen, T. Nakao, R. F. Bromdan, M. Greenberg, R. Charney, M. Menegus, M. Johnson, R. Grose, R. Frame, E. C. Hu, H. Choi and S. Safyer, "Early Postoperative Angiographic Assesment of Radial Artery Grafts Used for Coronary Artery Bypass Grafting," The Journal of Thoracic and Cardiovascular Surgery, Vol. 111, No. 6, 1996, pp. 1208-1214. http://dx.doi.org/10.1016/S0022-5223(96)70223-4

[3] F. D. da Costa, I. A. da Costa, R. Poffo, D. Abuchaim, R. Gaspar, L. Garcia and D. L. Faraco, "Myocardial Revascularisation with the Radial Artery: A Clinical and Angiographic Study,” The Annals of Thoracic Surgery, Vol. 62, No. 2, 1996, pp. 475-479. http://dx.doi.org/10.1016/0003-4975(96)00311-6

[4] C. A. Dietl and C. H. Benoit, "Radial Artery Graft for Coronary Revascularisation: Technical Considerations,” The Annals of Thoracic Surgery, Vol. 60, 1995, pp. 102-109.

[5] P. Pola, M. Serrichio, R. Flore, E. Manasse, A. Favuzzi and G. F. Possati, "Safe Removal of the Radial Artery for Myocardial Revascularisation: A Doppler Study to Prevent İschemic Complications to the Hand,” The Journal of Thoracic and Cardiovascular Surgery, Vol. 112, No. 3, 1996, pp. 737-744.

http://dx.doi.org/10.1016/S0022-5223(96)70060-0

[6] Ruengsakulrach, M. Brooks, D. L. Hare, U. Gordon and B. F. Buxton, "Preoperative Veluation of Hand Circulation by Means of Doppler Ultrasonography and ModifiedAllen Test," The Journal of Thoracic and Cardiovascular Surgery, Vol. 121, No. 3, 2001, pp. 526-531. http://dx.doi.org/10.1067/mtc.2001.112468
[7] O. F. Dogan, M. Karcaaltincaba, U. Duman, D. Akata, A. Besim and E. Boke, "Assessment of the Radial Artery and Hand Circulation by Computed Tomography Angiography: A Pilot Study,” Heart Surgery Forum, Vol. 8, No. 1, 2005, pp. 28-33.

http://dx.doi.org/10.1532/HSF98.20041042

[8] A. Nicolasi, P. Parsons, L. L. Pohl, R. A. Cimbria and G. N. Olinger, "Increased İncidence of Radial Artery Calcification in Patients with Diabetes Mellitus," Journal of Surgical Research, Vol. 102, No. 1, 2002, pp. 1-5. http://dx.doi.org/10.1006/jsre.2001.6303

[9] J. Nunoo-Mensah, “An Unexpected Complication after Harvesting the Radial Artery for Coronary Artery Bypass Grafting," The Annals of Thoracic Surgery, Vol. 66, No. 3, 1998, pp. 929-931. http://dx.doi.org/10.1016/S0003-4975(98)00559-1

[10] T. Shigematsu, T. Kono, K. Satoh, K. Yokoyama, T. Yoshida and K. Shirai, "Phosphate Overload Accelerates Vascular Calcium Depositin in End Stage Renal Disease Patients," Nephrology Dialysis Transplantation, Vol. 18, No. III, 2003, pp. 86-89.

[11] E. Kaufer, M. F. Stephen, F. Rosemary and F. B. Richard, "Pathology of the Radial Artery and Internal Thorasic Arteries Used as Coronary Artery Bypass Grafts,” The Annals of Thoracic Surgery, Vol. 63, No. 4, 1967, pp. 11181122. http://dx.doi.org/10.1016/S0003-4975(96)01393-8

[12] S. M. Kane-ToddHall, S. P. Taggart, H. Clements-Jewery and D. E. Roskell, "Pre-Existing Vascular Disease in the Radial Artery and Other Coronary Artery Bypass Conduits," European Journal of Medical Research, Vol. 26, No. 41, 1999, pp. 11-14.

[13] P. Muntner, J. Coresh, M. J. Klag, et al., "History of Myocardial İnfarction and Stroke among İncident and Stage Renal Disease Cases and Population Based Controls: An Analyses of Shared Risk Factors," American Journal of Kidney Diseases, Vol. 40, No. 2, 2002, pp. 2330. http://dx.doi.org/10.1053/ajkd.2002.34515

[14] M. R. Davies and K. A. Hruska, "Pathopysiological Mechanisms of Vascular Calcificaation in end Stage Renal Disease,” Kidney International, Vol. 60, 2001, pp. 472-479. http://dx.doi.org/10.1046/j.1523-1755.2001.060002472.x

[15] L. F. Olsson, R. Odselius, E. Ribbe and J. Hegbrant, "Evidence of Calcium Phosphate Depositions," American Journal of Kidney Diseases, Vol. 38, No. 2, 2001, pp. 377-383. http://dx.doi.org/10.1053/ajkd.2001.26104

[16] J. Gade, J. Aabech and R. I. Hansen, "The Upper Arm Arterio-Venous Fistula-An Alternative for Vascular Access in Haemodialysis," Scandinavian Journal of Urology and Nephrology, Vol. 29, No. 2, 1995, pp. 121-124. http://dx.doi.org/10.3109/00365599509180550

[17] R. Gradzki, R. K. Dhingra, F. K. Port, E. Roys, W. F. Weitzel and J. M. Messana, "Use of ACE İnhibitors İs Associated with Prolonged Survival of Arteriovenous Grafts," American Journal of Kidney Diseases, Vol. 38, No. 6, 2001, pp. 1240-1244. http://dx.doi.org/10.1053/ajkd.2001.29220 